\title{
The Role of MRI and Nerve Root Biopsy in the Diagnosis of Neurosarcoidosis
}

\author{
Fraser G.A. Moore, Frederick Andermann, John Richardson, \\ Donatella Tampieri and Robert Giaccone
}

\begin{abstract}
Objectives: Neurological involvement occurs in 5-15\% of patients with sarcoidosis and isolated "neurosarcoidosis" occurs in less than $1 \%$ of all cases. Classical clinical presentations have been described, such as bilateral facial palsy, but often the disease presents insidiously with varied signs and symptoms. We present a patient who required biopsy of a lumbar nerve root for diagnosis of chronic, progressive neurosarcoidosis and review the literature with an emphasis on diagnosis. Methods: We have reviewed a patient who presented with signs and symptoms related to infiltration of her meninges and nerve roots by sarcoidosis. All pertinent history and physical information was taken from interviews with the patient and review of her chart. Laboratory, radiographic, and pathological investigations are presented. Results and conclusions: A high index of suspicion is required for the diagnosis of neurosarcoidosis. Gadolinium-enhanced MRI is useful but the findings are often nonspecific, and there should be a low threshold for biopsy whenever the diagnosis is considered.
\end{abstract}

RÉSUMÉ: Le rôle de la RMN et de la biopsie nerveuse dans le diagnostic de la neurosarcoïdose. Objectifs: Cinq à $15 \%$ des patients atteints de sarcoïdose ont une atteinte neurologique et moins de un pour cent des patients ont une "neurosarcoïdose". Des modes de présentation clinique classiques ont été décrits, telle la paralysie faciale bilatérale, mais souvent la maladie commence insidieusement par des signes et des symptômes variés. Nous présentons le cas d'un patient chez qui on a dû avoir recours à une biopsie d'une racine nerveuse lombaire pour poser le diagnostic de neurosarcoïdose progressive chronique et nous revoyons la littérature en mettant l'emphase sur le diagnostic. Méthodes: Nous avons revu le cas d'un patient qui avait consulté pour des signes et des symptômes en relation avec une infiltration des méninges et de racines nerveuses par la sarcoïdose. Toute l'information pertinente sur l'histoire et l'examen physique a été extraite d'entrevues avec la patiente et de son dossier médical. Les investigations biochimiques, radiologiques et anatomopathologiques sont présentées. Résultats et Conclusions: Le diagnostic de neurosarcoïdose requiert un fort soupçon que cette maladie soit en cause. La RMN avec rehaussement par le gadolinium est utile, mais les constatations sont souvent non spécifiques. Quand ce diagnostic est considéré, on ne doit pas hésiter à procéder à une biopsie.

Can. J. Neurol. Sci. 2001; 28: 349-353

Sarcoidosis is a chronic, granulomatous disease of unknown etiology that most commonly presents with asymptomatic hilar adenopathy. Virtually any organ system can be involved. In approximately $5-15 \%$ of cases there are neurological manifestations. ${ }^{1-8}$ In less than $1 \%$ of all cases of sarcoidosis, ${ }^{1,5}$ or $3-17 \%$ of those with neurological manifestations, ${ }^{1,3,5}$ involvement is restricted to the nervous system (isolated "neurosarcoidosis"). In some instances, the diagnosis of neurosarcoidosis is suggested by a classical clinical presentation such as bilateral facial nerve palsy ${ }^{1}$ but it may involve any part of the nervous system. A variety of investigations have been suggested as being very useful for diagnosis ${ }^{1,3-5}$ but, in many cases, neurosarcoidosis is an extremely difficult diagnosis to establish.

We present a 58-year-old woman with chronic, progressive involvement of cranial and spinal nerves in which nerve root biopsy was required for diagnosis of neurosarcoidosis. This emphasizes the importance of a high index of suspicion for the disease in patients with chronic neurological symptoms and the need to aggressively pursue the diagnosis once suspected.

\section{Patient PResentation}

A 58-year-old, right-handed woman was admitted to hospital in February, 2000, for investigation of chronic neurological complaints that

From the Department of Neurology, Montreal Neurological Institute and Hospital, McGill University, Montreal, Quebec, Canada.

ReCEIVEd APRIL 6, 2001. ACCEPTED IN FINAL FORM June 25, 2001.

Reprint requests to: Frederick Andermann, Department of Neurology, McGill

University, Room 127, Montreal Neurological Institute, 3801 University St., Montreal, Quebec H3A 2B4 Canada 
included transient cranial nerve palsies, lower extremity weakness and paraesthesias, and bowel and bladder incontinence. Multiple previous investigations had shown only a persistent CSF pleocytosis (Table).

In 1992, the patient had a sub-acute onset of bilateral sensorineural hearing loss, dizziness when standing, and a sensation of poor balance (worse in the dark). She began using a cane and hearing aid. The hearing loss and balance problems continued to progress slowly. In 1994, she had acute onset of horizontal diplopia and was found to have a right VI nerve palsy, which resolved completely within two weeks.

In 1995, she began to be followed by one of the authors (RG). At that time, her neurological exam was normal, as were serum ACE, HIV serology, Lyme serology, ANA, VDRL, and CXR. A CT and MRI of her brain were remarkable only for sinusitis. The sinusitis was asymptomatic and was not investigated further. (She had been seen by an ENT specialist on several occasions at the time she began to develop hearing loss). An initial lumbar puncture (LP) (Table) showed elevated protein, decreased glucose, and lymphocytosis. This was confirmed on a repeat LP several days later. The CSF gram stain, bacterial culture, viral culture, fungal culture, cryptococcal antigen, VDRL, AFB, and p-ANCA were all negative. The CSF cytology was normal, and oligoclonal bands were absent. A third LP several months later continued to show lymphocytosis.

In 1996, she had an acute left lower motor neuron facial palsy. She received a two week course of oral prednisone, and the facial weakness resolved completely within three weeks. In 1997, she began to complain of mild leg weakness that manifested itself principally in difficulty going up stairs. There was associated decrease in sensation below her umbilicus, and occasional bowel and bladder incontinence. These new symptoms were all slowly progressive. In 1998, she had a possible nocturnal seizure and was started on both oral phenytoin and prednisone, with some improvement in her balance and no further seizures. In January 1999, as her prednisone was being tapered, she had acute worsening of her leg weakness. The prednisone was increased without improvement, and it was stopped in March 1999. Shortly afterward, she fractured her right hip after slipping in the bath. She recovered well and was stable until December when she was admitted to hospital with fever and worsened left leg weakness. She was treated with IV steroids and returned to her previous level of weakness within days.

Further investigations performed included another LP (in 1997, again showing negative cultures and AFB), a gallium scan in 1997, a myelogram in 1999, an MRI of the spine with gadolinium (T11 to S1) in January 2000, and an EMG with nerve conduction studies. All were normal.

At admission, she complained of severe leg weakness requiring a walker, decreased leg and sacral sensation, and complete lack of bowel and bladder control. Medications included phenytoin, maalox, colace, and ativan. Her past medical history included a hysterectomy, recurrent urinary tract infections, ocular laser treatment for a retinal hemorrhage due to a "blocked vein", and migraine headaches treated with naprosyn. There was no significant family history.

General physical examination was unremarkable, with no hepatosplenomegaly and no visible rash. Cranial nerves were normal apart from an inferior nasal quadrantanopsia in the right eye (secondary to the previous hemorrhage) and the bilateral hearing loss. Muscle bulk was normal, tone was slightly increased in the lower extremities, upper extremity power was normal, and lower extremity power was symmetrically decreased: iliopsoas was MRC grade 3/5, hamstrings were $4-/ 5$, gluteus medius and quadriceps were $4 / 5$, gastrocnemius was $4+/ 5$, and tibialis anterior and hip adductors were $5 / 5$. Muscle stretch reflexes were symmetrically brisk in the upper extremities, brisk in the right lower extremity, the left knee jerk was reduced, the left ankle jerk was absent, the right plantar response was flexor and the left, extensor. Coordination was normal. Vibration and joint position sensation were normal, there was clear loss of pinprick and temperature sensation on the medial right ankle and the perianal region, and decreased rectal sensation and tone. She required assistance to stand and walk.

Complete blood count, serum electrolytes, AST, ALT, alkaline phosphatase, LDH, Ca2+, ESR, ACE, and serum protein electrophoresis were normal. C-reactive protein $(7.9 \mathrm{mg} / \mathrm{l}$, normal < 5) and GGT (69 $\mathrm{U} / \mathrm{L}$, normal $<40$ ) were slightly elevated. C-ANCA, rheumatoid factor, and anti-nuclear antibody were normal. Lumbar puncture revealed an opening pressure of 12.5, with results similar to previous (Table). CSF cytology showed morphologically normal lymphocytes. CSF bacterial and TB cultures, India ink stain, viral PCR (for HSV, CMV, and EBV), VDRL, and ACE were all negative, and B2-microglobulin was increased $(6.54 \mathrm{mg} / \mathrm{L}$, normal approx. $<1.2 \mathrm{mg} / \mathrm{L})$. EMG and nerve conduction studies were consistent with multiple radiculopathies in both upper and lower extremities. An MRI of the brain and spinal cord showed gadolinium enhancement of multiple cranial nerves, the basal meninges, the spinal meninges and roots, and areas of the spinal cord (Figures 1-3). CXR, CT scan of the chest, ultrasound of the abdomen and pelvis, and gallium scan did not reveal disease outside of the nervous system. A PPD skin test was negative. A biopsy of dorsal lumbar nerve roots revealed noncaseating granulomas infiltrating the nerves (Figure 4). A diagnosis of neurosarcoidosis was made.

Treatment was begun with oral imuran and intravenous methylprednisolone ( 1 gram twice per week), and the patient returned home. Three months later (June 2000), after receiving in-patient physiotherapy five days per week for the previous month, she and her husband both felt she had improved. She could walk well with a walker, and take four or five steps without it. She no longer needed laxatives and was performing intermittent bladder catheterization. She felt her sensation was improved. On neurological exam she had improved her lower extremity power by one MRC grade (e.g. 4- to 4) in each of the weak muscles. Her reflexes were unchanged. She continued on the same treatment, and three months later (September 2000) her power had again improved by one grade and she could climb stairs using hand rails. Her weekly dose of methylprednisolone was gradually decreased to $250 \mathrm{mg}$ per week, but she complained of burning pains in her left arm and leg that resolved when the dose was increased to $500 \mathrm{mg}$. When seen recently (February 2001) she reported improved sensation on her trunk, a return of bladder sensation and slowly improving mobility.

Table: Lumbar Puncture Results






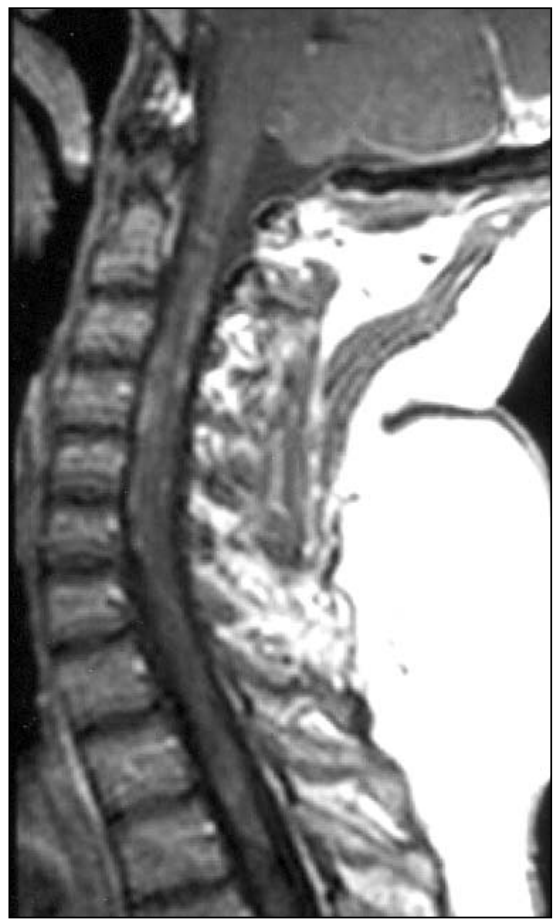

Figure 1: Sagittal T1-weighted MRI with gadolinium of the cervico-thoracic spine, demonstrating diffuse areas of enhancement both within and on the surface of the spinal cord. (Note that similar findings, as well as enhancement of the spinal roots, were seen in the lumbar region. The images were of poorer quality and are therefore not shown).

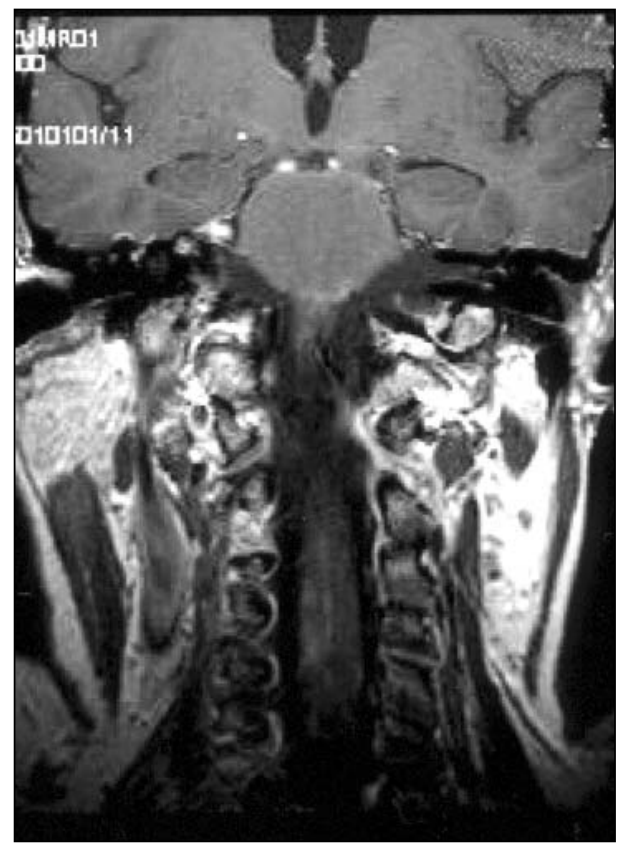

Figure 3: Coronal T1-weighted MRI of the brain with gadolinium, again demonstrating high signal intensity of the right fifth cranial nerve.

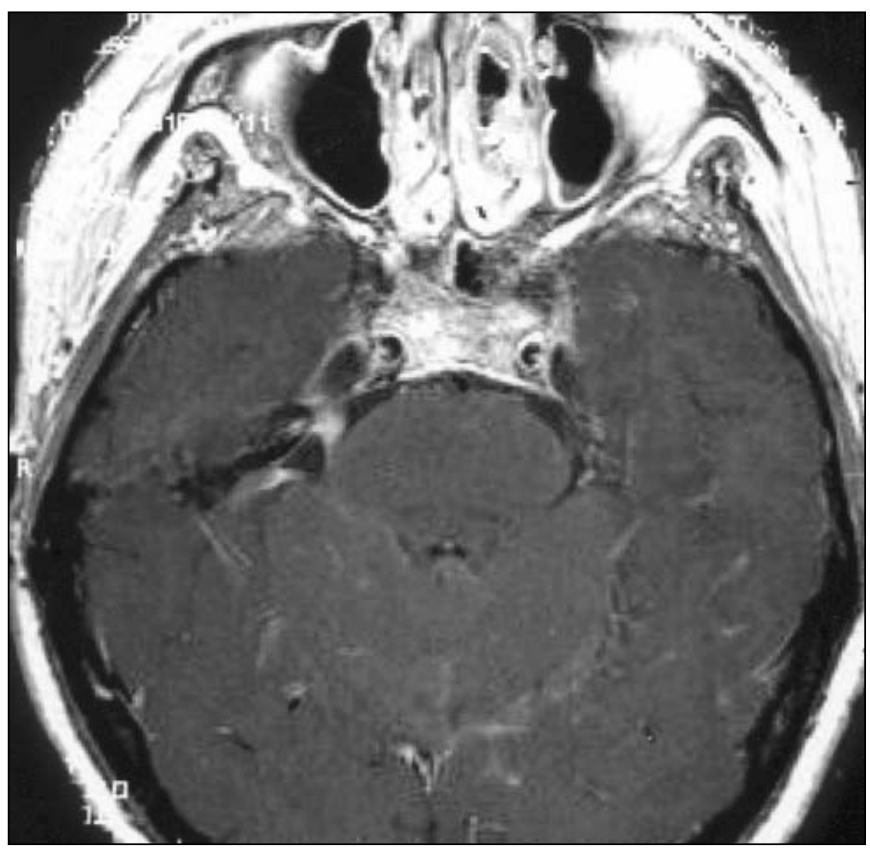

Figure 2: Axial T1-weighted MRI of the brain with gadolinium, demonstrating diffuse enhancement around the pons including the right fifth cranial nerve.

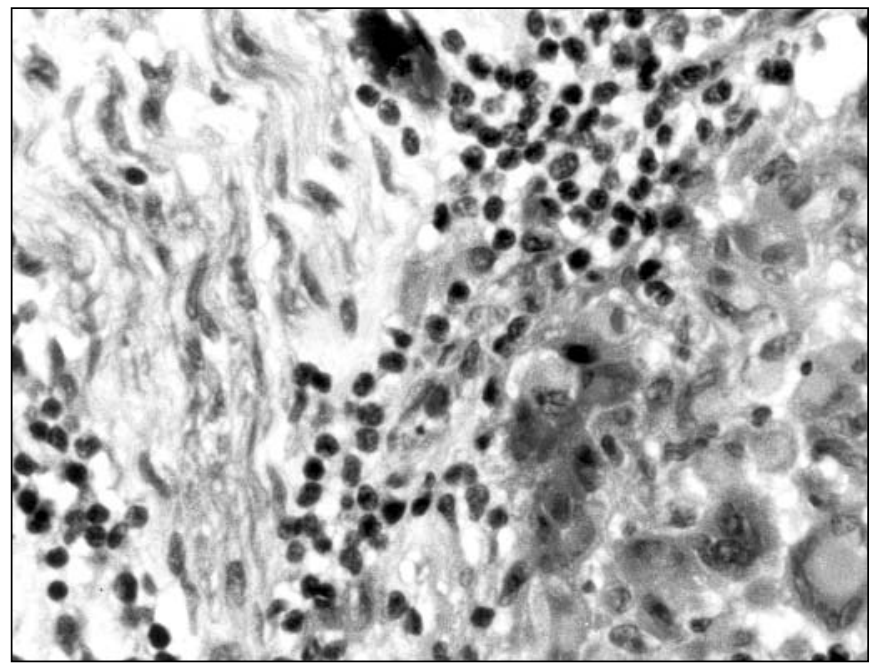

Figure 4: Histological section of biopsy specimen from dorsal lumbar nerve root. To the left of the figure the axons of the root can be seen. The remainder of the image is composed of a granuloma, at the center of which can be seen several giant cells. 


\section{Discussion}

Neurological symptoms are a rare manifestation of sarcoidosis, occurring in $5-15 \%$ of patients. ${ }^{1-8}$ Isolated "neurosarcoidosis" (with only the nervous system involved) is even rarer, occurring in less than $1 \%$ of all cases ${ }^{1,5}$ and $3-17 \%$ of those with neurological involvement. ${ }^{1,3,5}$ Manifestations include meningitis, seizures, mass lesions, diabetes insipidus, cranial nerve palsies, myelopathy, neuropathy and myopathy. ${ }^{3-6}$ Isolated involvement of the cauda equina and conus medullaris has been described but is felt to be rare. ${ }^{7-9}$ Our patient presented with signs and symptoms suggesting chronic, basal meningitis and later developed chronic, progressive leg weakness, numbness, and bowel and bladder disturbance likely caused by the granulomatous infiltration found at biopsy. The slowly progressive nature of her disease is consistent with both the reports of cauda equina involvement and isolated neurosarcoidosis in general. ${ }^{5,8}$ Sarcoidosis should therefore be suspected in patients presenting with chronic neurological dysfunction. Given the increasing number of case reports, it may not be as rare as once thought.

In suspected neurosarcoidosis, systemic manifestations should be looked for aggressively to confirm the diagnosis. ${ }^{1,4,5}$ In our patient, CXR, chest CT, and gallium scan were all normal. ${ }^{18}$ FDG PET scanning has also been used to detect systemic sarcoidosis. ${ }^{10,11}$ However, the resolution for lesions less than $10 \mathrm{~mm}$ in size is poor ${ }^{11}$ and therefore it is unlikely that the meningeal and nerve root infiltration seen in our patient would have been positive if ${ }^{18}$ FDG PET scanning had been done. Others have argued that the nonspecificity of increased systemic uptake of ${ }^{18} \mathrm{FDG}$, combined with the limited availability and cost of PET scanning, make its use in diagnosing sarcoidosis limited. ${ }^{12}$

Serum ACE levels are abnormally high in up to $80 \%$ of cases of systemic sarcoidosis, ${ }^{4,7}$ although some authors question its usefulness for diagnosis. ${ }^{6}$ It was normal in our patient and, in fact, is rarely useful for isolated neurosarcoidosis. ${ }^{7}$ Measurement of CSF ACE levels has been proposed as a diagnostic test, ${ }^{4,5,7}$ but recently the utility of this has been questioned. ${ }^{13}$ It was normal in our patient. Other CSF findings include elevated protein, pleocytosis, normal or decreased glucose, and elevated B2microglobulin. All are nonspecific and highly variable in neurosarcoidosis. ${ }^{4}$

Historically, the injection of antigen prepared from the liver or spleen of patients with sarcoidosis (the Kveim test) has been used for diagnosis but it is not well-standardized, nor commercially available. ${ }^{6,14}$ More recently, the Mantoux test site biopsy has been proposed as an alternative. ${ }^{14} \mathrm{~A}$ standard forearm skin Mantoux test is done and is typically negative, but skin biopsy of the site at a later date may reveal noncaseating granulomas. Steroid therapy may interfere with this, however, and there are no case series reported that have used this technique. Neither the Kveim test nor the Mantoux test site biopsy were done in our patient.

MRI can be suggestive of the diagnosis, with high sensitivity and specificity reported by some authors. ${ }^{15-17}$ Use of gadolinium greatly increases the sensitivity, in particular for meningeal disease. ${ }^{15,18}$ MRI was eventually positive in our patient, but earlier scans (including one of the spine with gadolinium enhancement) had been negative. Other authors have emphasized that the MRI appearance is nonspecific, ${ }^{7,8}$ especially of the spinal cord, ${ }^{17}$ and may be negative even in cases of histologically proven sarcoidosis with clear neurological dysfunction. ${ }^{16}$ In the most recent large series of sarcoidosis with nervous system involvement only 24 of 37 patients studies with MRI had positive findings. ${ }^{3}$ The authors speculated that this was because of treatment, and changes in the MRI findings of CNS sarcoidosis in response to steroid treatment are well described (especially loss of gadolinium enhancement ${ }^{15,16,19}$ ), however, the temporal relation between MRI and treatment for their patients was not presented. Finally, the MRI case series in sarcoidosis with nervous system involvement have included few patients with isolated CNS involvement.

Such a tremendous variety of MRI findings have been described (meningeal and cranial/spinal root enhancement, white matter lesions, parenchymal masses, hypothalamic lesions etc.) that, in the absence of systemic evidence of sarcoidosis, the diagnosis may not be considered even with an abnormal MRI. Clearly, none of the above tests are diagnostic of neurosarcoidosis. Therefore, the most important element in diagnosis is a high index of suspicion. Patients with chronic, slowly progressive neurological dysfunction in which sarcoidosis is suspected will often require biopsy for definitive diagnosis, ${ }^{7-9}$ as ours did.

Treatment and prognosis for such patients is controversial. Most authors favor prolonged treatment with corticosteroids ${ }^{1-6}$ but there is only anecdotal evidence to support this. Immunosuppression with azathioprine, ${ }^{20}$ cyclosporine, ${ }^{4,20,21}$ methotrexate, ${ }^{3,4,20}$ and cyclophosphamide ${ }^{3,20}$ are tried as adjuvant therapy if the response to steroids is poor or side effects develop. Others use these agents only in severely ill patients. ${ }^{5}$ Radiation treatment has also been tried. ${ }^{4,5}$ We elected to treat with weekly intravenous methylprednisolone and oral imuran, in the hope of maximizing treatment while avoiding the side effects of oral steroids and the stronger immunosuppressants. Prognosis with treatment is felt to be good, particularly if begun early, and up to $50 \%$ of patients may remit., ${ }^{1,4-6}$ With longer duration of symptoms, such as in our patient, the chance of complete recovery is much less ${ }^{8}$ but significant improvement can be obtained. Physiotherapy can also play an important role in helping patients regain some degree of functional independence, ${ }^{9}$ and our patient certainly seemed to benefit. Both treatment and prognosis clearly need to be individualized.

In summary, our case is exceptional in the duration and variety of neurological complaints. It points out the need for both a high index of suspicion for the diagnosis of neurosarcoidosis and for aggressive investigations to pursue this. In patients with suspected involvement of the cauda equina careful MRI examination with, and without, gadolinium should be performed, and there should be a low threshold for consideration of biopsy.

\section{REFERENCES}

1. Stern BJ, Krumholz A, Johns C, Scott P, Nissim J. Sarcoidosis and its neurological manifestations. Arch Neurol 1985;42:909-917.

2. Delaney P. Neurological manifestations of sarcoidosis: review of the literature, with a report of 23 cases. Ann Intern Med 1977;87:336345.

3. Lower EE, Broderick JP, Brott TG, Baughman RP. Diagnosis and management of neurological sarcoidosis. Arch Int Med 1997; 157:1864-1868.

4. Scott TF. Neurosarcoidosis: Progress and clinical aspects. 
Neurology 1993;43:8-12.

5. Chapelon C, Ziza JM, Piette JC, et al. Neurosarcoidosis: signs, course and treatment in 35 confirmed cases. Medicine 1990;69(5):261-276.

6. Silberberg DH. Sarcoidosis of the nervous system. In: Aminoff MJ ed. Neurology and General Medicine. New York: Churchill Livingstone, 1995.

7. Jallo GI, Zagzag D, Lee M, et al. Intraspinal sarcoidosis: diagnosis and management. Surg Neurol 1997;48:514-521.

8. Zajicek J. Sarcoidosis of the cauda equina: a report of three cases. $\mathbf{J}$ Neurol 1990;237:424-426.

9. Ku A, Lachmann E, Tunkel R, Nagler W. Neurosarcoidosis of the conus medullaris and cauda equina presenting as paraparesis: case report and literature review. Paraplegia 1996;34:116-120.

10. Lewis PJ, Salama A. Uptake of Fluorine-18-Fluorodeoxyglucose in sarcoidosis. J Nucl Med 1994;35:1647-1649.

11. Yamada Y, Uchida Y, Tatsumi K, et al. Fluorine-18-Fluorodeoxyglucose and Carbon-11-Methionine evaluation of lymphadenopathy in sarcoidosis. J Nucl Med 1998;39:1160-1166.

12. Alavi A, Buchpiguel CA, Loessner A. Is there a role for FDG PET imaging in the management of patients with sarcoidosis? J Nucl Med 1994;35:1650-1652.

13. Dale JC, O'Brien JF. Determination of angiotensin-converting enzyme levels in cerebrospinal fluid is not a useful test for the diagnosis of neurosarcoidosis. Mayo Clin Proc 1999;74:535.
14. Mankodi AK, Desai AD, Mathur RS, Poncha FF. Diagnostic role of Mantoux test site biopsy in neurosarcoidosis. Neurology 1998;51:1216-1218.

15. Sherman JL, Stern BJ. Sarcoidosis of the CNS: comparison of unenhanced and enhanced MR images. Am J Radiol 1990;155:1293-1301.

16. Lexa FJ, Grossman RI. MR of sarcoidosis in the head and spine: spectrum of manifestations and radiographic response to steroid therapy. Am J Neuroradiol 1994;15:973-982.

17. Christoforidis GA, Spickler EM, Recio MV, Mehta BM. MR of CNS sarcoidosis: correlation of imaging features to clinical symptoms and response to treatment. Am J Neuroradiol 1999;20:655-669.

18. Junger SS, Stern BJ, Levine SR, et al. Intramedullary spinal sarcoidosis: clinical and magnetic imaging characteristics. Neurology 1993;43:333-337.

19. Dumas JL, Valeyre D, Chapelon-Abric C, et al. Central nervous system sarcoidosis: follow-up at MR imaging during steroid therapy. Radiology 2000;214:411-420.

20. Agbogu BN, Setn BJ, Sewell C, Yang G. Therapeutic considerations in patients with refractory neurosarcoidosis. Arch Neurol 1995;52:875-879.

21. Stern BJ, Schonfeld SA, Sewell C, et al. The treatment of neurosarcoidosis with cyclosporine. Arch Neurol 1992;49:10651072 . 